\title{
Theoretical Prediction of the Mass Flow Rates in the Bubble Pump
}

\author{
Author: B.Gurevich* \\ Shamoon College of Engineering, Department of Mechanical Engineering, Ashdod, Israel \\ E-mail: bellagu@sce.ac.il
}

Received 23 October 2019, Revised 21 November 2019, Accepted 25 November 2019

\begin{abstract}
The bubble pump is an essential part of diffusion absorption cooling systems where heating, pumping of the binary solution, and the separation occur. The existing theoretical models of the bubble pump were initially developed for air lift pumps where neither heating nor separation occurs. Thus, the experimental results for a bubble pump did not correlate well with the theoretical models. Empirical values were suggested in some of the models; however, their values varied from one system to another and could not be predicted analytically. In this work a modified model based on mass, energy, momentum, and heat balances is presented with the utilization of the drift flux model with laminar flow assumption. The objective of the work was to develop a theoretical model that can predict the mass flow rates for a given geometry, operational conditions and applied heat. Unlike previous works for the first time the applied heat is expressed in the model. The suggested model fits better with the experimental results than the previous models.
\end{abstract}

\section{Keywords: Diffusion absorption cooling systems, bubble pump, drift flux model.}

\section{Introduction}

Diffusion absorption cooling systems are heat driven and contain no moving parts such as a compressor or a pump. The working fluid is a mixture of a coolant and a solvent together with an inert gas. The main part of such systems is the bubble pump where heating, pumping of the solution, and the separation occur. The bubble pump is a heated tube (length $\mathrm{L}$ and diameter D) connecting two reservoirs (Fig. 1). Initially, the level of the liquid in the lift tube is the same as in the lower reservoir $(\mathrm{H})$. Heat is supplied at the bottom of the bubble pump (generator) and causes the gaseous coolant bubbles to flow up the lifting tube. The heat causes reduction in the bulk density of the fluid in the tube in comparison to that in the lower reservoir, which creates a buoyancy effect. At the upper reservoir (the separator), the gas is separated from the liquid solution. In diffusion absorption refrigeration (DAR) systems the bubble pump is responsible for the circulation of the binary solution containing a coolant (usually a refrigerant) and an absorbent (usually an organic solution). Coefficient of performance (COP) values of DAR systems are low and in the range of 0.1-0.15.

DARs were first presented by Platen and Munters in 1921 and later by Einstein and Szilard in 1930. Despite the DAR potential, there are not many works in the literature that focus on the bubble pump. A more covered topic in the literature is the air-lift pump that operates with the same principles as vapor-lift pumps with the exception of air being injected to increase the buoyancy of the fluid instead of bubbles forming from liquid vaporization.

Stenning and Martin [1] were the first to develop an analytical model for an airlift pump. In their model onedimensional mass and momentum equations together with the basic equations of two-phase flow, taking into consideration the effects of friction and slip between the gas and liquid phases (drift flux model), were used. Liquid volume flow rates were plotted versus air volume flow rates for various submergence ratios $(\mathrm{H} / \mathrm{L})$. A comparison with experimental work was carried out, and the authors predicted that the theory of one-dimensional flow is sufficient for the analysis of airlift pump performance.

Delano [2] studied theoretically and experimentally the vapor bubble pump used in the Einstein System. The model of Stenning and Martin [1] was first modified to analyze the performance of the bubble pump. All the supplied heat was assumed to evaporate the water without any heat loss. The velocity of the bubbles was assumed to be constant. Delano [2] also used a constant value of slip factor (the ratio of velocities of the vapor and the water). Later, Koyfman et al. [3] showed that the velocities of water and vapor change with the operating parameters. Since single-phase flow was assumed, the gas void fraction was avoided. The model results indicated that the mass flow rate of the liquid varied with heat input and tube diameter. Instead of using the drift flux model to find the void fraction slip value, $S$ was assigned.

Sathe [4] studied theoretically and experimentally the bubble pump performance assuming that the flow in the vertical lift tube was laminar and using $\mathrm{K}$ adjustable parameter to account for losses other than friction in the tube. The mass flow rate of vapor increased linearly with the heat input while the mass flow rate of the pumped liquid first increased, reached a maximum value, and then decreased with the increase in the heat input. A larger diameter pump tube was assumed to be advantageous. However, increasing the diameter with a fixed liquid flow eventually caused transition from the assumed slug flow [5] to bubbly flow.

Shihab and Morad [6] performed a theoretical and experimental study on the vapor bubble pump. The theoretical prediction of the pumping capacities was lower than the experimental results for all values of tube diameter and 
submergence ratio. A new K-factor equation was introduced to correlate the theoretical result with the experimental data.

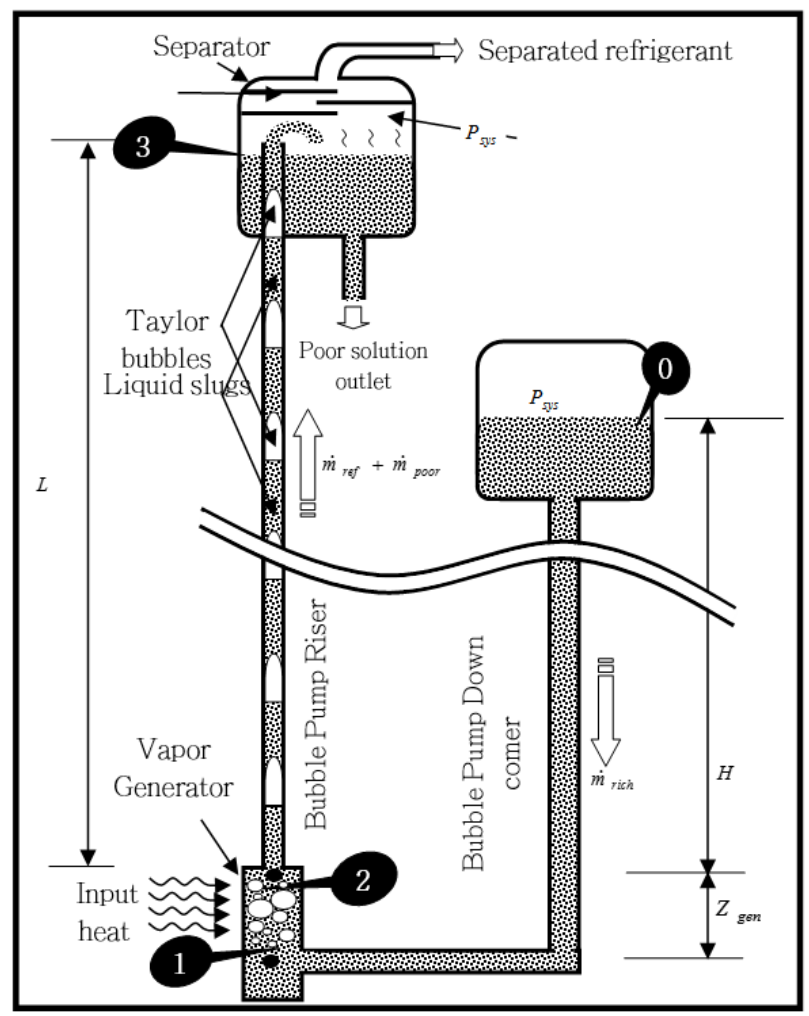

Figure 1. Schematic drawing of the bubble pump.

The work of Gurevich et al. [7] experimentally studied the performance of three parallel bubble pumps operating with a solution of R134a-DMAC. The results revealed that in comparison to a single lift tube, the use of two or three parallel lift tubes at optimal operating conditions (heat input and concentration of the refrigerant) could double or triple, correspondingly, the quantity of desorbed refrigerant. There was not a good correlation with the theoretical model of Delano [2].

A mathematical model that was developed by Pffaf et al. [8] was based on the manometer principle. A transparent experimental rig was built to visualize the flow behavior and to validate the analytical model. The experimental results were lower than the predicted results.

Aman et al. [9] presented a theoretical model using the drift flux model and assuming a turbulent flow regime. The submergence ratio was presented as a function of dimensionless Froude numbers and frictional pressure drop. Heat losses from the lift tube were considered and were calculated based on an empirical correlation [10]. The theoretical results were correlated with the experimental results of water.

The current models are not accurate enough to predict the experimental results. Empirical values are widely used; however, these values vary from one system to another and cannot be generalized. Also, the heat input that drives the whole system is not presented in the models.

\section{Theoretical Model}

The theoretical model is based on mass balances, energy balances, and one-dimensional momentum balances with additional models. $\dot{m}_{\text {rich }}$ is the mass flow the rich solution.
Referring to Figure 1, applying the mass balance equation from point 0 to point 1 :

$$
\dot{m}_{o}=\dot{m}_{1}=\dot{m}_{\text {rich }}
$$

where $\dot{m}_{\text {rich }}$ containing the solvent and the gas (coolant or refrigerant), assuming that its value doesn't change along section $0-1$.

$P_{0}$ is the system pressure, the sub-index DC refers to the down comer (down comer diameter $D_{D C}$ ). Heights $\mathrm{H}$ and $Z_{\text {gen }}$ are shown in Figure 1. Appling the Bernoulli equation from the liquid surface $(0)$ at the down comer to point (1) taking into consideration head loss due to friction:

$$
\begin{aligned}
& \frac{P_{0}}{\rho_{0} g}+\frac{V_{0}^{2}}{2 g}+\left(H+Z_{g e n}\right)= \\
& =\frac{P_{1}}{\rho_{1} g}+\frac{V_{1}^{2}}{2 g}+f \frac{\left(H+Z_{g e n}\right)}{D_{D C}} \frac{V_{1}^{2}}{2 g}
\end{aligned}
$$

Assuming that the level of the solution in the reservoir is maintained constant $\left(V_{0}=0\right)$. The densities in points (0) and (1) are assumed to be equal to the density of the rich solution, $\rho_{\text {rich }}\left(\rho_{o}=\rho_{1}=\rho_{\text {rich }}\right)$ which is in liquid phase, but consists of two fluids, the refrigerant and the solvent. The velocity of the fluid in section (1) can be expressed as:

$$
V_{1}=\frac{\dot{m}_{\text {rich }}}{\rho_{\text {rich }} \cdot A_{\text {gen }}}
$$

Note that $A_{g e n}$ is the cross section of the generator where heating occurs. Thus the pressure difference between sections (1) and (0) is:

$$
\begin{aligned}
& P_{0}-P_{1}=\frac{\dot{m}_{\text {rich }}{ }^{2}}{2 \rho_{\text {rich }} \cdot A_{\text {gen }}{ }^{2}}\left[1+f\left(\frac{H+Z_{\text {gen }}}{D_{D C}}\right)\right]- \\
& +\rho_{\text {rich }} g\left(H+Z_{\text {gen }}\right)
\end{aligned}
$$

Mass and momentum conservation equations are applied for the generator. Point (1) is the entrance to the generator and point (2) is the outlet of the generator. Let us assume that at the outlet of the generator there is a two-phase flow consisting of the evaporated refrigerant as the gas phase $\dot{m}_{r e f}$ and the poor solution containing $\dot{m}_{\text {poor }}$ the solvent with residues of gas as the liquid phase. The sub-index gen refers to the generator.

The density along the generator is consistent of the gas density and the liquid (poor solution containing the solvent and refrigerant that was not separated due to heating) density, thus it is described with TP sub index-two phase.

Consequently, the pressure drop along the generator neglecting friction can be expressed as:

$P_{1}-P_{2}=\frac{\dot{m}_{\text {rich }}}{A_{\text {gen }}} \cdot\left(V_{2}-V_{1}\right)$

The area of interest is the lifting tube. Therefore, mass and momentum balances are applied between points (2) and (3). Two phase solution flows upward in the lifting tube. Let us assume that the mass flow rates of the refrigerant, $\dot{m}_{r e f}$, and the poor solution, $\dot{m}_{\text {poor }}$, are constant along the lift tube (points (2) and (3)). The sub-index LT refers to the lift tube. $\dot{m}_{2}=\dot{m}_{3}=\dot{m}_{\text {rich }}=\dot{m}_{\text {poor }}+\dot{m}_{\text {ref }}$

At point (3) the pressure is assumed to be system pressure $\left(P_{\mathrm{o}}\right)$. The overall pressure drop along the lift tube (length $\mathrm{L}$, area 
$A_{L T}, D_{L T}$ the inner diameter of the lift tube where two phase occurs) can be expressed as a function of the acceleration pressure drop, $\Lambda P_{a c c}$, gravitational pressure drop, $\Lambda P_{\text {grav }}$, and frictional pressure drop, $\Lambda P_{\text {fric }}$.

$$
\begin{aligned}
& P_{2}-P_{3}=\underbrace{\frac{\dot{m}_{\text {rich }}}{A_{L T}} \cdot\left(V_{3}-V_{2}\right)}_{\Delta P_{a c c}}+\underbrace{\rho_{T P} \cdot g \cdot L}_{\Delta P_{\text {grav }}}+ \\
& +\underbrace{f_{T P} \rho_{T P} \frac{1}{2}\left(\frac{\dot{m}_{\text {rich }}}{A_{L T}}\right)^{2} \frac{L}{D_{L T}}}_{\Delta P_{\text {fric }}}
\end{aligned}
$$

Assuming that the velocities of the two phases $V_{2}$ and $V_{3}$ along the lift tubes do not vary, the acceleration pressure drop can be neglected $\left(\Delta P_{a c c}=0\right)$. The expression for the pressure drop can be written in the following manner:

$$
P_{2}-P_{3}=\Delta P_{\text {grav }}+\Delta P_{\text {fric }}
$$

The gravitational term $\left(\Lambda P_{\text {grav }}\right)$ represents the weight of the refrigerant (gas) and the solvent (liquid) mixture column in the lifting tube.

$\Delta P_{\text {grav }}=\rho_{T P} \cdot g \cdot L$

The density of the two phase solution is calculated by using the drift flux model. The drift-flux approach is based on the consideration of two fluids as a mixture of the phases in which properties are represented as an average of the properties of the two phases. The slug mixture density, $\rho_{T P}$ ,is defined as the average of the liquid and gas phase densities weighted by the void fraction $\alpha$ :

$\rho_{T P}=(1-\alpha) \rho_{L}+\alpha \rho_{G}$

The one-dimensional drift flux model assumes that the two-phase flow properties do not change across the pipe cross-section, and hence yield the values that are averaged across the pipe cross-section for any given axial pipe location. The void fraction in this model is a function of the phase superficial velocity $V_{G S}, C_{0}$ the distribution parameter, $V_{m}$ the average mixture velocity, and $V_{G M}$ the local drift velocity:

$$
\alpha=\frac{V_{G S}}{C_{0} V_{m}+V_{G M}}
$$

The superficial velocity is defined as gas volumetric flowrate, $Q_{G}$, divided by the pipe cross-section A:

$$
V_{G S}=\frac{Q_{G}}{A}
$$

The average mixture velocity is defined as:

$$
V_{m}=\frac{Q_{L}+Q_{G}}{A}
$$

There are several correlations to calculate the distribution parameter and the local drift velocity. For laminar flows $C_{0} \approx 2$.[10-14]. According to Bonnecaze et al. [15] the local drift velocity is:

$$
V_{G M}=0.35 \sqrt{\frac{g D\left(\rho_{L}-\rho_{G}\right)}{\rho_{L}}}
$$

Sub-indexes of the density are $\mathrm{L}$ for liquid and $\mathrm{G}$ for gas. In the present model the liquid refers to the poor solution and the gas refers to the refrigerant.
The friction pressure drop of the two phase solution in the lift tube is:

$\Delta P_{\text {fric }}=f_{T P} \frac{V_{m}^{2} \rho_{T P}}{2} \frac{L}{D_{L T}}$

Assuming laminar flow:

$$
f_{T P}=\frac{64}{\operatorname{Re}_{m}}=\frac{64}{\frac{V_{m} \rho_{T P} D_{L T}}{\mu_{T P}}}
$$

The Reynolds number is defined as:

$\operatorname{Re}_{m}=\frac{V_{m} \rho_{T P} D_{L T}}{\mu_{T P}}$

$V_{m}$ is the total average superficial velocity, $\rho_{T P}$ and $\mu_{T P}$ are the density and the dynamic viscosity of the two-phase solution. By summing Eqs. (4), (5), and (8):

$\frac{\dot{m}_{\text {rich }}^{2}}{2 \rho_{\text {rich }} \cdot A_{D C}^{2}}\left[1+f\left(\frac{H+Z_{\text {gen }}}{D_{D C}}\right)\right]-\rho_{\text {rich }} g\left(H+Z_{g e n}\right)+$

$+\frac{\dot{m}_{\text {rich }}}{A_{\text {gen }}} \cdot\left(V_{2}-V_{1}\right)+\rho_{T P} \cdot g \cdot Z_{\text {gen }}+$

$+\rho_{T P} \cdot g \cdot L+f_{T P} \frac{V_{m}^{2} \rho_{T P}}{2} \frac{L}{D}=0$

The density and the dynamic viscosity of the binary solution (poor solution and rich solution) at equilibrium with respect to temperature $T$ and $\xi$, the concentration is given by [16]:

$\rho=\sum_{j=0}^{3} \sum_{i=0}^{3} \rho_{i, j} \cdot \xi^{i} \cdot T^{j}$

$\ln (\mu)=\sum_{j=0}^{3} \sum_{i=0}^{3} \mu_{i, j} \cdot \xi^{i} \cdot T^{j}$

Thus, the submergence ratio can be expressed as:

$$
\begin{aligned}
& \frac{\left(H+Z_{g e n}\right)}{\left(Z_{\text {gen }}+L\right)}=\frac{\dot{m}_{\text {rich }}{ }^{2}}{\rho_{\text {rich }} \cdot g \cdot\left(Z_{\text {gen }}+L\right)} \\
& \cdot\left[\frac{1}{2 \rho_{\text {rich }} \cdot A_{D C}^{2}}+\frac{1}{A_{g e n}{ }^{2}} \cdot\left(\frac{1}{\rho_{T P}}-\frac{1}{\rho_{\text {rich }}}\right)\right]+ \\
& +\frac{\rho_{T P}}{\rho_{\text {rich }}}
\end{aligned}
$$

For a fixed submergence ratio there are two unknown parameters, $\dot{m}_{\text {rich }}$ the mass flow rate of the rich solution and $\dot{m}_{r e f}$ the mass flow rate of the separated coolant (expressed in the void fraction calculation).

We express the mass flow rate of the refrigerant as a function of the inlet heat input. To do so we apply heat balance on the generator with the assumption that the inlet temperature to the generator is equal to the reservoir temperature. The supplied heat input $\dot{Q}$ heats the rich solution in the generator, increasing its temperature from the equilibrium temperature $T_{0}$ to the generator temperature $T_{\text {gen }}$.

$$
\dot{Q}=\dot{m}_{\text {rich }} \cdot \bar{c}_{p}\left(T_{\text {gen }}-T_{0}\right)
$$

The heat capacity of the solution can be considered as the average of the heat capacity of the rich solution at the inlet to the generator (point (1)) and the heat capacity of the poor solution leaving the generator (point (2)). 
The heat capacity values were based on Yokozeki's [17] experimental results. Thus the model can be rewritten:

$$
\begin{aligned}
& \frac{\left(H+Z_{\text {gen }}\right)}{\left(Z_{\text {gen }}+L\right)}=\frac{\dot{m}_{\text {rich }}{ }^{2}}{\rho_{\text {rich }} \cdot g \cdot\left(Z_{\text {gen }}+L\right)} \\
& \cdot\left[\frac{1}{2 \rho_{\text {rich }} \cdot A_{D C}{ }^{2}}+\frac{1}{A_{\text {gen }}{ }^{2}} \cdot\left(\frac{1}{\rho_{T P}}-\frac{1}{\rho_{\text {rich }}}\right)\right]+ \\
& +\frac{\rho_{T P}}{\rho_{\text {rich }}}
\end{aligned}
$$

Note that the geometrical and operational conditions are known and the only variable in the model is $\rho_{T P}$, which is a function of the void fraction, which is a function of refrigerant and poor solutions mass flowrates. By applying mass conservation (Eq. 6), the void fraction can be expressed as a function of the rich mass flow rate and poor mass flow rate. Rich mass flow rate can be expressed as a function of the applied heat. Thus from the model one can calculate the poor solution mass flow rate and the refrigerant mass flow rate.

\section{Experimental Results}

The model was compared with the experimental results of Gurevich et al. [7]. Based on the presented model flow rates of the rich, poor and refrigerant were calculated and plotted as a function of the applied heat in comparison to the experimental results (Fig.2). Rich and poor solutions mass flow rates depend on the amount of applied heat, whereas the refrigerant solution is almost independent of the amounts of the applied heat. Experimental results of the rich and poor solutions show a reasonable correlation with the model results.

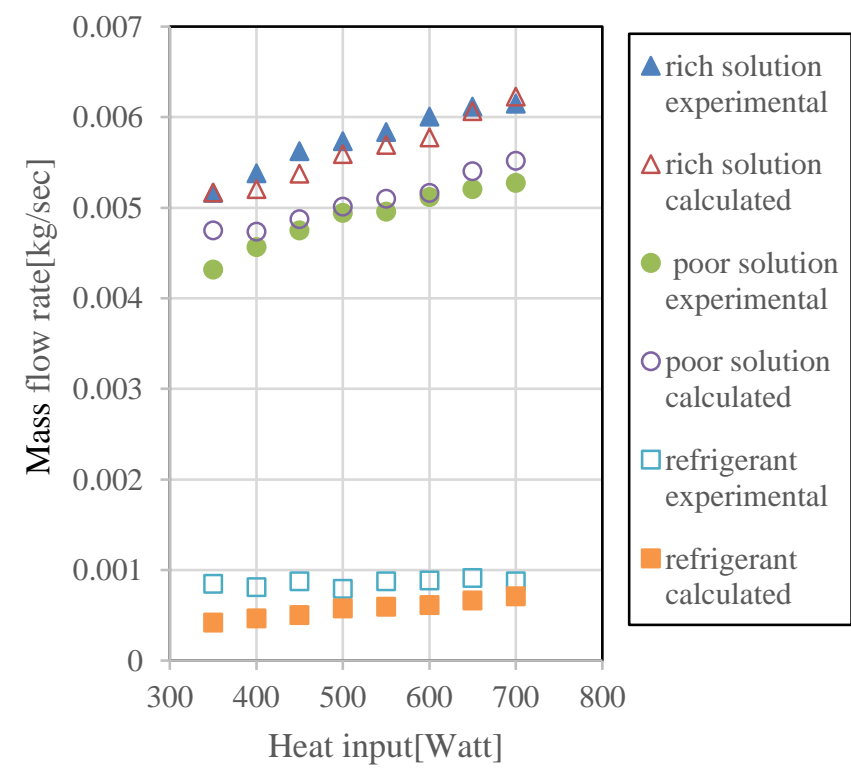

Figure 2. Experimental and calculated mass flow rates based on the model in Eq. (24) vs. the experimental results of Gurevich et al. [7], $H / L=0.7$.

It can be seen from Figure 2 that the higher the flow rates the more accurate the model is. The assumption is that low flow rates, there is less probability for slug flow in the lifting tubes, thus the lifting effect is no efficient [2].

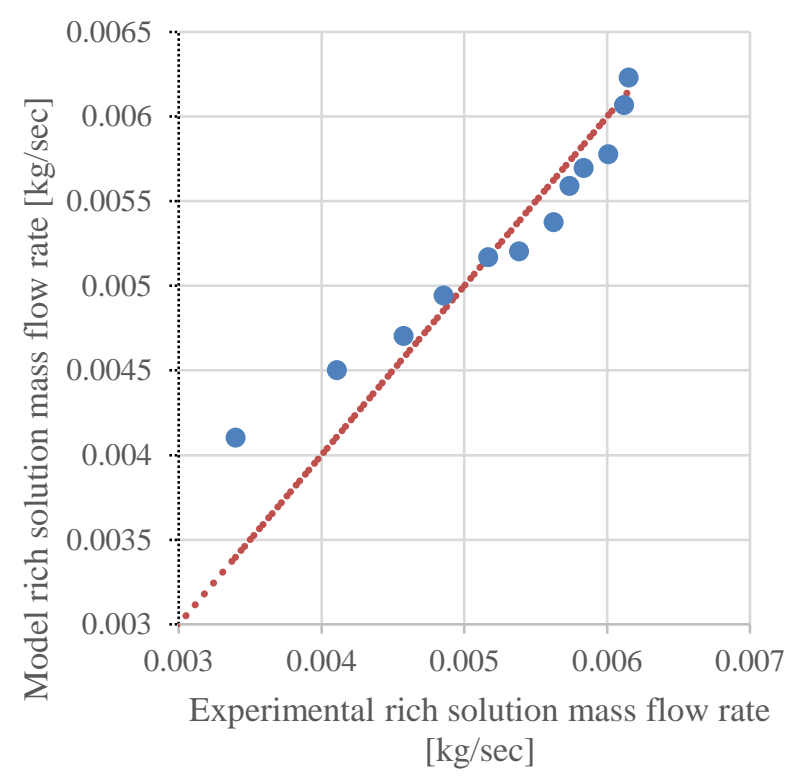

Fig. 3: Calculated rich solution mass flow rate based on the model in Eq. (24) vs. the experimental results Gurevich et al.[7], $h / L=0.7$.

The difference can be seen more clearly in Figure 3. The increase in the flow rates occurs with an increase in the heat input. For low flow rates (less than 150 Watts per pipe and 450 Watts for three parallel lifting tubes [7]) the experimental results do no correlate well with the model due to the inefficient lifting.

It can be seen from Figure 4 that the current model that uses the drift flux model with the assumption of laminar flow fits more accurately to the experimental results

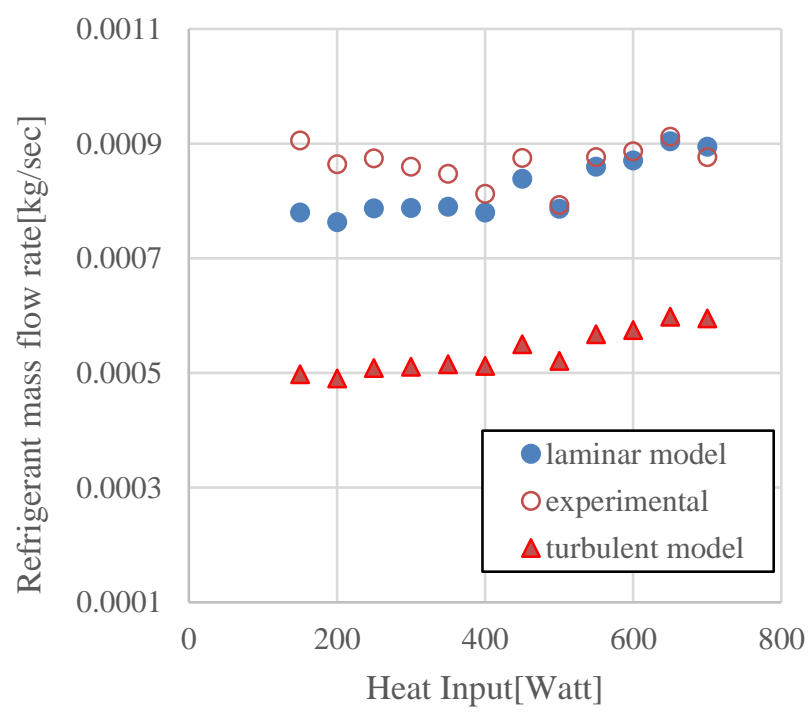

Figure 4. Separated mass flow rate of the refrigerant $v$ s. heat input for experimental results, model with laminar flow assumption, and model with turbulent flow assumption, $h / L=0.7$.

The results in Table 1 confirm that the assumption of laminar flow was correct for the current system when referring to the liquid and the mixture flow regimes. 
Table 1. Reynolds numbers of the two-phase mixture, the poor solution, the rich solution, and the gas as a function of input heat.

\begin{tabular}{ccccc}
\hline & \multicolumn{4}{c}{ Reynolds Number } \\
\hline Heat[Watt] & Mixture & $\begin{array}{c}\text { Poor } \\
\text { Solution }\end{array}$ & $\begin{array}{c}\text { Rich } \\
\text { solution }\end{array}$ & Gas \\
\hline 150 & 286.9 & 131.8 & 94.92 & 3536 \\
\hline 200 & 412.9 & 204.6 & 133.6 & 3342 \\
\hline 250 & 558.7 & 284.0 & 174.7 & 3344 \\
\hline 300 & 671.9 & 347.8 & 202.8 & 3264 \\
\hline 350 & 798.3 & 420.3 & 232.7 & 3193 \\
\hline 400 & 969.2 & 519.4 & 275 & 3017 \\
\hline 450 & 1133.0 & 605.2 & 313.9 & 3216 \\
\hline 500 & 1282.0 & 702.0 & 350.2 & 2882 \\
\hline 550 & 1420.0 & 768.1 & 377.3 & 3159 \\
\hline 600 & 1445.0 & 783.9 & 368.6 & 3236 \\
\hline 650 & 1614.0 & 874.5 & 407.0 & 3290 \\
\hline 700 & 1822.0 & 996.3 & 454.9 & 3114 \\
\hline & & & &
\end{tabular}

\section{Conclusions}

A theoretical model based on Stenning and Martin's model $^{1}$ was presented. The presented model is based on the drift flux model together with laminar flow assumption. Experimental results show that the flow of the poor solution (the liquid phase) is indeed laminar. The model allows prediction of the amounts of the separated refrigerant for a certain submergence value within the values of heat input where lifting occurs. In addition, the present model correlates between the amount of the applied heat and the rich solution mass flow rate. It is important to state that the previous models were developed for adiabatic all-water lift tubes where heat did not play any significant role. The present model correlates the geometrical and the operational parameters of the bubble pump and fits better to the experimental results.

\begin{tabular}{|c|c|c|}
\hline \multicolumn{2}{|c|}{ Nomenclature } & \multirow[b]{2}{*}{ Pipe cross section } \\
\hline$A$ & $\left\lfloor m^{2}\right\rfloor$ & \\
\hline$C_{o}$ & & Distribution parameter \\
\hline$c_{p}$ & $\mathrm{~kg}$ & Heat capacity \\
\hline & $\mathrm{kg} \cdot{ }^{o} \mathrm{C}$ & \\
\hline$D$ & {$[m]$} & Pipe diameter \\
\hline$f$ & & Friction coefficient \\
\hline$g$ & {$\left[\frac{m}{\sec ^{2}}\right]$} & Gravity \\
\hline$H$ & {$[m]$} & Motive head \\
\hline$L$ & {$[m]$} & Length of the lift tube \\
\hline$\dot{m}$ & {$\left[\frac{k g}{\mathrm{sec}}\right]$} & Mass flow rate \\
\hline$P$ & {$[\mathrm{~Pa}]$} & Pressure \\
\hline$Q$ & {$\left[\frac{m^{3}}{\mathrm{sec}}\right]$} & Volumetric flow rate \\
\hline
\end{tabular}

\begin{tabular}{|c|c|c|}
\hline$\dot{Q}$ & {$[$ Watt $]$} & Heat \\
\hline$R e$ & & Reynolds number \\
\hline$T$ & {$\left[{ }^{\circ} \mathrm{C}\right],[K]$} & Temperature \\
\hline$V$ & {$\left[\frac{m}{\mathrm{sec}}\right]$} & velocity \\
\hline$Z$ & {$[m]$} & Generator height \\
\hline $\begin{array}{l}\text { Greek } \\
\quad \alpha\end{array}$ & ymbols & Void fraction \\
\hline$\mu$ & {$\left[\frac{\mathrm{kg}}{\mathrm{m} \cdot \mathrm{sec}}\right]$} & Viscosity \\
\hline$\xi$ & & Mass concentration \\
\hline$\rho$ & {$\left[\frac{\mathrm{kg}}{\mathrm{m}^{3}}\right]$} & Density \\
\hline \multicolumn{2}{|c|}{ Subscripts } & Points $0,1,2,3$ \\
\hline $\mathrm{DC}$ & \multicolumn{2}{|c|}{ Down comer } \\
\hline$f$ & \multicolumn{2}{|c|}{ Friction } \\
\hline G & \multicolumn{2}{|c|}{ Gas } \\
\hline gen & \multicolumn{2}{|c|}{ Generator } \\
\hline $\mathrm{L}$ & \multicolumn{2}{|c|}{ Liquid } \\
\hline LT & \multicolumn{2}{|c|}{ Lift tube } \\
\hline $\mathrm{m}$ & \multicolumn{2}{|c|}{ mixture } \\
\hline poor & \multicolumn{2}{|c|}{ Poor solution } \\
\hline ref & \multicolumn{2}{|c|}{ Refrigerant } \\
\hline rich & \multicolumn{2}{|c|}{ Rich solution } \\
\hline sys & \multicolumn{2}{|c|}{ System } \\
\hline $\mathrm{TP}$ & \multicolumn{2}{|c|}{ Two phase } \\
\hline
\end{tabular}

\section{References:}

[1] A. H. Stenning and C. . Martin, "An Analytical and Experimental Study of Air-Lift Pump Performance," Journal of Engineering for Gas Turbines and Power, vol. 90, no. 2, pp. 106-112, Apr. 1968.

[2] A. Delano, "Design Analysis of the Einstein Refrigeration Cycle," Ph.D. Thesis, Georgia Institute of Technology, USA, 1998.

[3] A. Koyfman, M. Jelinek, A. Levy, and I. Borde, “An experimental investigation of bubble pump performance for diffusion absorption refrigeration system with organic working fluids," Applied Thermal Engineering, vol. 23, no. 15, pp. 1881-1894, Oct. 2003.

[4] A. Sathe, "Experimental and Theoretical Studies on a Bubble Pump for a Diffusion-Absorption Refrigeration System," University of Stuttgart, Germany, 2001.

[5] D. Chisholm, Two-phase flow in pipelines and heat exchangers. London; New York: G. Godwin in association with Institution of Chemical Engineers, 1983.

[6] A. S. W. Shihab and A. M. A. Morad, "Experimental Investigation of Water Vapor- Bubble pump Characteristics and its Mathematical Model 
Reconstruction,” Eng. \& Tech. Jolurna, vol. 30, no. 11, pp. 1870-1885, 2012.

[7] B. Gurevich, M. Jelinek, A. Levy, and I. Borde, "Performance of a set of parallel bubble pumps operating with a binary solution of R134a-DMAC," Applied Thermal Engineering, vol. 75, pp. 724-730, Jan. 2015.

[8] M. Pffaf, R. Saravanan, M. P. Maiya, and S. S. Murthy, "Studies on bubble pump for a water-lithium bromide vapour absorption refrigerator," Int. J. Refrigeration, vol. 21, no. 6, pp. 452-562, 1998.

[9] J. Aman, P. Henshaw, and D. S.-K. Ting, "Performance characterization of a bubble pump for vapor absorption refrigeration systems," International Journal of Refrigeration, vol. 85, pp. 58-69, Jan. 2018.

[10] T. Katasuhara and T. Kazama, "Heat Transfer in TwoPhase Flow of Mixtures of Air and Water. 2nd Report Vertical Channel," Transection of JSME, vol. 24, pp. $552-558,1958$

[11] M. Ishii and T. Hibiki, "One-Dimensional Drift-Flux Model," in Thermo-Fluid Dynamics of Two-Phase Flow, Boston, MA: Springer US, 2006, pp. 381-418.

[12] R. Collins, F. F. D. Moraes, J. F. Davidson, and D. Harrison, "The motion of a large gas bubble rising through liquid flowing in a tube," Journal of Fluid Mechanics, vol. 89, no. 03, p. 497, Dec. 1978.

[13] J. R. Grace and R. Clift, "Dependence of slug rise velocity on tube Reynolds number in vertical gas-liquid flow," Chemical Engineering Science, vol. 34, no. 11, pp. 1348-1350, 1979.

[14] K. H. Bendiksen, "On the motion of long bubbles in vertical tubes," International Journal of Multiphase Flow, vol. 11, no. 6, pp. 797-812, Nov. 1985.

[15] R. H. Bonnecaze, W. Erskine, and E. J. Greskovich, "Holdup and pressure drop for two-phase slug flow in inclined pipelines," AIChE Journal, vol. 17, no. 5, pp. 1109-1113, Sep. 1971.

[16] I. Borde, M. Jelinek, and N. C. Daltrophe, "Development of Advanced Absorption Systems Driven by Low Temperature Heat Sources," Int. J. Refrigeration, vol. 31, pp. 521-530, 1993.

[17] A. Yokozeki, "Theoretical performances of various refrigerant-absorbent pairs in a vapor-absorption refrigeration cycle by the use of equations of state," Applied Energy, vol. 80, no. 4, pp. 383-399, Apr. 2005. 